Itinéraires Itinéraires

Littérature, textes, cultures

2018-1| 2018

Le manifeste à travers les arts : devenirs d'un genre indiscipliné

\title{
Bâtir le manifeste : de La Città Nuova d'Antonio Sant'Elia au manifeste architectural contemporain
}

Building the Manifesto: From Antonio Sant'Elia's La Città Nuova to the Contemporary Architectural Manifesto

\section{Viviana Birolli}

\section{OpenEdition}

\section{Journals}

Édition électronique

URL : http://journals.openedition.org/itineraires/4223

DOI : $10.4000 /$ itineraires.4223

ISSN : 2427-920X

Éditeur

Pléiade

\section{Référence électronique}

Viviana Birolli, « Bâtir le manifeste : de La Città Nuova d'Antonio Sant'Elia au manifeste architectural contemporain », Itinéraires [En ligne], 2018-1 | 2018, mis en ligne le 15 septembre 2018, consulté le 16 mai 2020. URL : http://journals.openedition.org/itineraires/4223 ; DOI : https://doi.org/10.4000/ itineraires. 4223

Ce document a été généré automatiquement le 16 mai 2020.

\section{cc) (†) $\odot$}

Itinéraires est mis à disposition selon les termes de la licence Creative Commons Attribution - Pas d'Utilisation Commerciale - Pas de Modification 4.0 International. 


\title{
Bâtir le manifeste : de La Città Nuova d'Antonio Sant'Elia au manifeste architectural contemporain
}

\author{
Building the Manifesto: From Antonio Sant'Elia's La Città Nuova to the \\ Contemporary Architectural Manifesto
}

Viviana Birolli

1 Si nous voulions comparer cet article à un édifice, il se construirait au carrefour de deux projets de bâtiment: le premier questionnant une histoire des manifestes en architecture, le second approfondissant la construction du manifeste en tant que genre et objet théorique, à la lumière notamment des ambiguïtés de statut et de définition soulevées par les évolutions récentes de cette pratique discursive.

2 En effet, en dépit de la «mort » du genre proclamée pendant les années $1980^{1}$, on n’a jamais cessé d'écrire des manifestes. Récemment, cette continuité du phénomène manifestaire a fait l'objet d'un regain d'intérêt de la part de la critique : en témoignent les numéros monographiques des revues Francofonia ${ }^{2}$, Lignes ${ }^{3}$, Études Littéraires (Birolli et Tjell 2013), Marges (Jaudon, Birolli et al. 2015) et l'ouvrage After the Manifesto... (Buckley 2015), pour ce qui concerne respectivement les domaines de la littérature, des arts plastiques et de l'architecture. Les questions qui structurent ces études portent à la fois sur la nature, les fonctions et la définition des gestes manifestaires récents, caractérisés par des formes hybrides détournant souvent les visées des manifestes avant-gardistes. L'idée d'un anachronisme du manifeste est-elle encore valable aujourd'hui, du moins pour ce qui concerne le domaine de l'architecture? Dans quelle mesure et à quelles conditions est-il légitime de faire évoluer la définition du manifeste, au vu de l'éventail de formes très large dont font preuve les exemples contemporains du genre?

3 Afin de développer ce double plan historique et théorique, nous questionnerons la spécificité du manifeste en architecture à travers une analyse de ses évolutions formelles et définitionnelles. Une mise en miroir de deux graphiques issus du corpus de 
manifestes répertoriés dans la base de données Manart sera le point de départ pour formuler et illustrer ce double propos.

Fig 1. Manifestes en architecture, 1903-2013

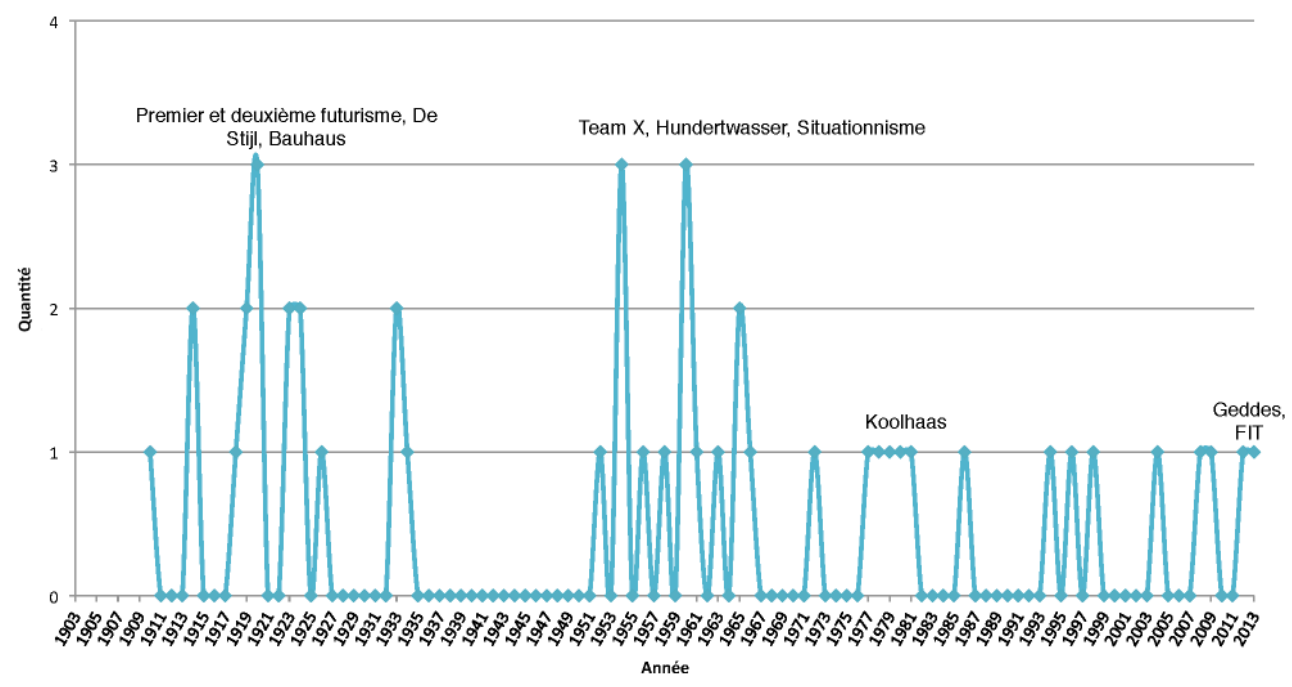

Base Manart, février 2015

Le premier graphique retrace les évolutions du manifeste en architecture entre 1903 et 2013, s'appuyant sur un corpus de 43 manifestes recensés dans Manart pour cette discipline en février 2015. Photographie instantanée d'un recensement évolutif (Birolli et Bloomfield 2013 : 149-150), ce graphique prend en compte une base géographique très vaste (incluant à la fois l'Europe et les États-Unis) et comprend des manifestes au statut différent - titrés comme tels par leurs auteurs ou qualifiés a posteriori ainsi par la critique: des programmes d'école aux déclarations visant à une reconnaissance institutionnelle de la discipline, en passant par des projets utopiques.

Il permet néanmoins de dresser quelques constats sur la fortune, relativement faible, du manifeste en architecture : de 1910 à 2013, les pics de production ne dépassent pas les trois occurrences par an. Les publications se concentrent autour de deux moments clés : le premier couvre un arc chronologique qui va des années 1910 à la moitié des années $1920^{4}$ - avec un bref regain d'intérêt au milieu des années 1930, correspondant aux exploits architecturaux du deuxième futurisme ${ }^{5}$; le second couvre une période qui va de la moitié des années 1950 aux premières années $1960^{6}$. Par la suite, le manifeste ne cessera d'émailler l'histoire de l'architecture, pendant les années 1970, puis de nouveau à partir du début du Xxi ${ }^{\mathrm{e}}$ siècle.

6 Les courbes dessinées par ces données correspondent assez fidèlement aux résultats d'une précédente enquête sur les développements du manifeste dans les arts plastiques (Bloomfield et Tjell 2013), recoupant les deux saisons des avant-gardes historiques et des «néo-avant-gardes» du début des années 1960. Pour ce qui concerne le destin contemporain du manifeste, ce graphique semble témoigner d'une continuité possible du phénomène, sans pour autant en décréter le retour en force. 
Fig. 2. Manifestes en architecture, 1903-2013

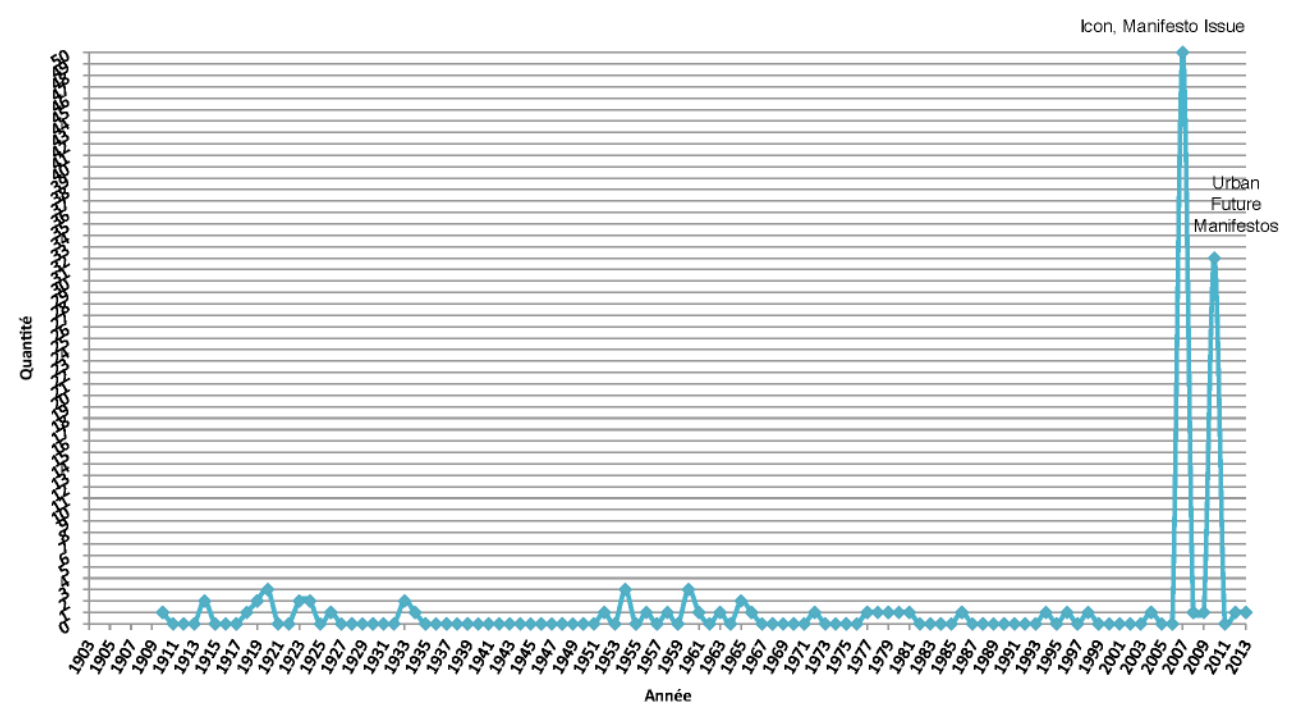

Base Manart (février 2015) après ajout de deux recueils manifestaires récents.

7 Le deuxième graphique se veut une simulation dotée d'une valeur heuristique et résulte de l'ajout, au corpus du premier graphique, de deux recueils manifestaires de la première décennie du $\mathrm{xxI}^{\mathrm{e}}$ siècle : le dossier «Fifty manifestos", publié par la revue d'architecture Icon en août 2007, et Urban Future Manifestos, édité en 2010 par le MAK Center de Los Angeles (Noever et Meyer 2010). Les quatre-vingt-deux manifestes rassemblés dans ces deux publications - cinquante dans la première, trente-deux dans la seconde - ne sembleraient laisser aucun doute quant au destin contemporain du genre : non seulement le manifeste n'est pas mort, mais on dirait qu'il n'a jamais été aussi vivant ${ }^{7}$.

8 En dépit de leurs divergences, les deux graphiques sont légitimes pour décrire une histoire du manifeste; simplement, ils ne se réfèrent pas au même objet. Comment, à quel prix et à quelles conditions le mot - «manifeste »- peut-il alors correspondre à la chose $^{8}$ - «le manifeste de » - selon l'un des plus éternels dilemmes sémiotiques ?

9 L'écart entre ces deux graphiques dessine en creux l'histoire d'un glissement ${ }^{9}$ qui s'est opéré au sein du manifeste, prenant d'abord la forme d'une fissure, puis d'une métamorphose (ou recyclage ?) affectant à la fois l'aspect du manifeste et sa définition. Faite d'allers-retours entre modalités de production et modalités de réception - volonté des auteurs et volonté des exégètes - cette histoire dessine une dynamique interprétative complexe nourrie par un questionnement simple. Qu'est-ce qui est manifeste par ses caractéristiques textuelles et qu'est-ce qui l'est devenu par des attributions critiques successives? Quel statut conférer aux nouvelles formes manifestaires qui redéfinissent le visage du genre depuis la seconde moitié $d u x^{e}$ siècle? Faut-il considérer comme une force d'évolution légitime l'élaboration de nouvelles définitions calquées sur de nouveaux manifestes? Pour démêler ces questions, nous observerons des exemples qui se situent aux pôles extrêmes du manifeste en architecture, par leur chronologie et leur forme :

- Le manifeste futuriste d'Antonio Sant'Elia (juillet 1914), largement reconnu comme l'un des idéaux-types du manifeste d'avant-garde en architecture, dont les caractères typiques mais 
aussi les marges d'ambiguïté soulignent quelques-unes des tensions qui ont le plus influencé l'histoire du manifeste dans ce domaine.

- Delirious New York. A Retroactive Manifesto for Manhattan de Rem Koolhaas (1978), considéré comme le premier diagnostic de la mort du manifeste et de sa renaissance en tant que discours « rétroactif ", et Learning from Las Vegas de Robert Venturi (1972). À la même époque où, dans les arts plastiques, l'on décrétait l'anachronisme du manifeste, en architecture la prise en compte des évolutions possibles de cette pratique discursive après la fin du modernisme a contribué à permettre son retour dans des formes métamorphosées.

- Enfin, les deux recueils qui font l'objet du deuxième graphique : deux cas qui mettent à mal toute tentative de définition qui aspirerait à embrasser les évolutions du manifeste le long d'un arc chronologique large. Rédigés sur commande pour paraître dans des recueils collectifs, ces manifestes ne pourraient être plus éloignés des modèles avant-gardistes.

L'hétérogénéité qui caractérise ces textes à l'intérieur du cadre commun imposé par leur titre - «manifeste »- fait de la détermination des conditions de possibilité et des formes d'une redéfinition du manifeste une question préliminaire pour toute étude de ses évolutions contemporaines. L'analyse de quelques-unes des étapes marquantes de l'histoire du manifeste en architecture permet de mettre l'accent sur la spécificité du développement du genre dans ce domaine et de souligner les tensions profondes qui influencent ses métamorphoses au fil du temps. De plus, ces évolutions du manifeste dessinent une histoire des rapports changeants entre l'architecture et sa théorie : d'un côté la ville, de l'autre son programme passé, présent ou futur.

\section{Le manifeste de l'architecture futuriste d'Antonio Sant'Elia : bâtir un manifeste}

11 Manifeste s'il en est, le texte signé par l'architecte italien Antonio Sant'Elia est canonique par son mode de publication et de diffusion: L'architettura futurista. Manifesto $^{10}$ est diffusé à Milan le 11 juillet 1914 en tant que tract de la Direction du mouvement futuriste (Caruso 1980) et sera par la suite publié à plusieurs reprises dans des revues futuristes ${ }^{11}$. Même s'il est signé seulement par Sant'Elia, ce tract se veut ainsi l'émanation d'une action collective et s'inscrit à plein titre dans le projet d'une «Reconstruction futuriste de l'univers ${ }^{12}$ ». Cette inscription collective ne s'exprime pas seulement sur le plan linguistique, mais aussi dans la mise en page et la hiérarchisation des informations. Selon la structure typique des manifestes futuristes, le texte s'ouvre ainsi par une partie polémique d'accusations contre l'Italie et contre toute l'architecture européenne, où se définit l'opposition entre le "nous » qui accuse et le " vous » contre lequel on se lance, le futur qu'on réclame comme présent et le présent que l'on veut reléguer dans le passé.

12 La maison et la ville futuriste - premiers mots marqués en gras dans le texte surgissent de l'état de crise brossé dans les premiers paragraphes comme une nécessité : «La maison et la ville spirituellement et matériellement nôtres [...] projection immédiate et fidèle de ce que nous sommes", seront des "machines gigantesques ", nouvelles " comme est nouveau notre état d'âme ». "L'architecture se détache de la tradition. On recommence à zéro, forcément ».

13 Le manifeste se poursuit en développant les détails de cette esthétique qui se veut aussi un art du construire et se conclut avec la classique liste de ce que le futurisme « combat 
et méprise» et de ce qu'il "proclame». À bas les «carnavalesques incrustations décoratives»-selon le dogme moderniste formulé en 1908 par Adolf Loos (1910). Longue vie au perpétuel renouveau des "maisons qui dureront moins que nous", au « goût du léger, du pratique, de tout ce qui est éphémère et rapide ». Vive l'architecture futuriste, architecture « du calcul, de l'audace téméraire et de la simplicité [...] du ciment béton, du fer, du verre, du carton, de la fibre textile ", mais aussi " art, à savoir synthèse, expression ".

14 Par rapport à d'autres manifestes futuristes, le texte de Sant'Elia se caractérise par une oscillation au niveau des déictiques - entre le «nous» des futuristes et le «je» de l'architecte - et par une section iconographique qui fait des dessins de la ville futuriste un manifeste, au même titre que le texte. La tension entre composants, textuel et iconographique, est du reste l'un des traits typiques du manifeste en architecture.

Mis à part ces spécificités, le manifeste de Sant'Elia correspond en tout point aux normes de cet «art d'écrire les manifestes » que Marinetti s'auto-attribuait et qui a profondément influencé la définition du manifeste d'avant-garde. Néanmoins, c'est au niveau des modalités de production que le manifeste signé par l'architecte de Côme révèle une marge d'ambiguïté : enrichi des ajouts de Marinetti et de son secrétaire Decio Cinti (Carrà 1981: 101), ce texte s'avère être le fruit d'un travail à plusieurs mains. Ces remaniements étaient courants dans le cadre de la « fabrique » manifestaire futuriste, dont Marinetti se voulait l'arbitre incontesté. Ici, toutefois, ces modifications font l'objet d'une polémique en juillet 1956, lorsque l'architecte Giovanni Bernasconi republie, dans Rivista tecnica della Svizzera italiana, le Messaggio rédigé en mai 1914 par Sant'Elia pour le catalogue d'une exposition du groupe Nuove Tendenze ${ }^{13}$.

Publié à peine deux mois avant le manifeste, ce Messaggio en est aussi proche dans le fond que différent dans la forme : l'objet de la polémique qui éclate à la lumière de ces contradictions devient dès lors l'intention de Sant'Elia d'adhérer sans réserve au futurisme ${ }^{14}$. Du point de vue de l'œuvre de Sant'Elia, la valeur de cette «question purement italienne » (Banham 1957 : 626) est moindre, d'autant que le groupe Nuove Tendenze se définissait lui-même comme « l'aile droite du futurisme ».

Du point de vue d'une analyse du manifeste, en revanche, cette confrontation n'est pas dépourvue d'intérêt: la plupart des ajouts de Marinetti concernent les passages qui font basculer le texte de Sant'Elia du régime de la déclaration programmatique à celui du manifeste ${ }^{15}$, et la posture (Meizoz 2007) de son auteur d'une attitude déclarative à une approche polémique de type manifestaire. Ce changement de statut du texte se répercute aussi sur la lecture des dessins: de libre représentation architecturale conçue pour une exposition d'art, La Città Nuova devient, par sa labellisation en manifeste, un projet réclamant une concrétisation aussi urgente que pratique.

18 Il s'agit là d'un glissement de statut riche en implications, si nous considérons qu'il a été l'une des conditio sine qua non du traitement « hagiographique " auquel Marinetti soumet la figure de Sant'Elia en $1930^{16}$, à l'occasion de la commémoration de sa mort prématurée, survenue au front le 10 octobre 1916 alors que l'architecte n'avait pas encore trente ans : la réévaluation critique de son œuvre est destinée à se déployer sous le signe d'une promesse brisée. Le manifeste et les projets de La Città Nuova permettent alors à Marinetti, par le caractère prétendument factuel de la langue manifestaire, de faire de Sant'Elia le prophète futuriste de la modernité architecturale. Toutefois, l'aura mythique de "grand constructeur » moderniste dont Marinetti a entouré l'architecte italien correspond mal aux commandes traditionnelles auxquelles ce dernier travaillait 
dans les dernières années de sa vie, ainsi qu'aux grandes difficultés posées par l'adaptation posthume de l'un de ses projets: gérée d'abord par le peintre Enrico Prampolini, ensuite par l'architecte Giuseppe Terragni entre 1926 et 1934, la traduction en monument aux morts de Côme d'une tour-phare dessinée par Sant'Elia en 1914 dut faire face aux ambiguïtés d'un projet où aucune mesure, aucun plan et aucune échelle n'étaient mentionnés. C'est donc moins dans ses projets que dans l'écart entre l'œuvre et le mythe façonné en partie par le manifeste que se situe la racine des polémiques qui ont rythmé la réception critique de l'architecte italien.

19 Texte prétendument transparent, le manifeste peut aussi s'avérer un document opaque ; source documentaire de première main, il peut également faire l'objet d'un aménagement intéressé et d'une mythographie dont l'impact historique ne doit pas être sous-estimé. Ainsi, au cœur d'un exemple canonique nous soulignons deux traits qui influenceront toute l'histoire du manifeste en architecture : premièrement, ce lien entre manifeste et programme qui est aussi au centre du recueil d'Ulrich Conrads. Si dans les arts plastiques l'identité du manifeste s'est construite à partir de modèles littéraires et politiques, le manifeste en architecture tend en effet à être perçu plutôt comme un prolongement des textes programmatiques rédigés par les groupes, les écoles et les personnalités qui rythment l'histoire de la discipline. Deuxièmement, ce décalage entre texte, dessin et traduction pratique, architecture sur papier et architecture bâtie que la critique reprochera souvent aux architectes modernistes, quand l'utopie deviendra, d'élan créatif et idéal qu'elle était, péché capital et échec on ne peut plus réel. Chez Sant'Elia, ces deux questions présentent des liens au cœur du manifeste, texte caractérisé par la capacité de "produire l'événement ${ }^{17}$ » et de faire basculer les utopies en programmes et ces derniers en projets, parfois au-delà des intentions de leurs auteurs.

\section{Le manifeste et le programme : réverbérations du manifeste}

Publié en 1964, le recueil d'Ulrich Conrads Programmes et manifestes de l'architecture du $\mathrm{XX}^{e}$ siècle reste à ce jour la seule anthologie consacrée à l'histoire du manifeste en architecture du début du XXe siècle (1903) au début des années 1960 (1963).

Comme son titre l'affirme, ce recueil ne fait pas la distinction entre programme et manifeste: ce dernier y est considéré comme l'une des déclinaisons du discours programmatique en architecture. La sélection des documents du recueil s'opère dès lors selon un critère de réception critique et historique : "N'ont été retenus que les textes qui, d'une part, constituent le début d'une tendance déterminée de la construction et qui, d'autre part, ont eu une influence déterminante sur le développement réel de l'architecture en Europe centrale " (Conrads [1964] $1996: 12)^{18}$.

C'est en appliquant ces critères de réception et d'efficacité historique que la sélection d'Ulrich Conrads finit par inclure beaucoup de textes qui font l'objet d'attributions manifestaires a posteriori, en dépit de leur origine déclarative ou programmatique. Un exemple pour tous, Cinq points d'une architecture nouvelle, texte célèbre de $1927^{19}$ où Le Corbusier annonce les cinq piliers de la nouvelle architecture (pilotis, toits-jardins, fenêtres en longueur, plans et façades libres). Unanimement reconnu comme le programme de l'architecture moderne, au fil du temps ce texte a souvent frôlé le 
manifeste sous la plume de la critique, selon des marges d'oscillation capricieuses qui vont de l'attribution des Cinq points au genre manifestaire, à son association au manifeste bâti qui en serait la traduction, à savoir la villa Savoye de Poissy.

Le basculement entre programme et manifeste évoqué pour Sant'Elia se prolonge ainsi, chez Conrads, dans un questionnement sur ce qui est perçu comme programme ou comme manifeste. Anna Boschetti le remarque bien :

[...] l'analyse de la notion de manifeste ne saurait s'en tenir à une approche lexicographique ni partir d'une définition. [...] Il convient donc de ne pas se borner à prendre en considération les textes présentés explicitement dès leur parution comme manifestes, mais la constellation plus ample des textes qui ont été perçus comme «manifestaires». (Boschetti $1980: 13$ )

S'appuyant sur ce terrain flou qui lie le manifeste à d'autres formes textuelles pouvant acquérir une valeur manifestaire, le recueil de Conrads permet dès lors d'amorcer une analyse du manifeste en tant que genre ${ }^{20}$ construit au croisement entre volonté des auteurs et des exégètes. Il s'agit là d'un terrain d'étude opaque, qui a en revanche l'avantage de bien saisir d'une part, un phénomène de réverbération des formules linguistiques et rhétoriques $d u$ manifeste vers d'autres formes textuelles $-d u$ programme au slogan; d'autre part, le poids de la réception dans la construction d'une histoire et d'un mythe du manifeste.

Dans cette dynamique de production générique (Schaeffer 1989: 79-82) par la réception, les traits textuels de chaque document se mélangent avec les péripéties de son historicisation, qui, à son tour, est souvent liée avec la fortune éditoriale. Les choix mêmes de Conrads ont sans doute joué un rôle dans la "manifestarisation" d'une partie des textes du recueil: si le manifeste façonne les lecteurs et les médias, ces derniers font souvent le manifeste. Du reste, Alice Kaplan avait déjà remarqué cette circularité entre manifeste et effet manifestaire: "Comme objets d'étude, les manifestes et les pamphlets affectent leur propre théorie » (1983: 75).

En même temps, le regard qu'Ulrich Conrads porte sur le manifeste dans l'introduction de son anthologie n'est pas flatteur: à la moitié des années 1960, cet architecte allemand salue la mort du manifeste comme le signe de la fin des pensées séparées et d'une pratique moderniste de l'architecture dont le défaut le plus grave serait la pénurie de preuves construites. Source de la plupart des polémiques qui ont accompagné la réévaluation de l'œuvre dessinée d'Antonio Sant'Elia, cette mise en question de la hiérarchie des rapports entre idée, représentation et pratique architecturale est aussi l'impulsion première de Delirious New York. A retroactive manifesto for Manhattan (Koolhaas 1978). La notion de manifeste rétroactif introduite par Rem Koolhaas dans cet ouvrage montre que l'évolution et la réception critique du manifeste en architecture débordent des cadres définitionnels traditionnels du genre: à partir de l'espace de pensée inauguré par sa forme rétroactive, le manifeste en architecture sera un objet appelé à se redéfinir, encore et encore.

\section{New York délire, Las Vegas enseigne : le manifeste rétroactif}

27 En dépit de son titre, Delirious New York. A retroactive manifesto for Manhattan n'a rien d'un manifeste au sens classique : là où le manifeste avant-gardiste se voulait un texte concis, «à lire sans lunettes », le texte de l'architecte néerlandais se présente sous la 
forme d'un livre de plus de 300 pages. Là où le manifeste se voulait une arme langagière, le style de Koolhaas ne craint pas la digression. La nouveauté introduite par cet ouvrage dans le genre manifestaire réside en revanche dans le rapport qu'il institue entre la ville et son programme : l'analyse du présent et les propositions pour le futur de Delirious New York découlent d'une archéologie de ce qui existe, selon une conception qui fait du présent le fruit d'un passé à réexaminer pour relancer un discours possible sur le futur. Les genèses « sans pères » et la conception linéaire progressive de l'histoire dont le manifeste moderniste était le théâtre ne pourraient être plus loin. Pourtant, la dynamique qui fait du texte de Koolhaas un manifeste puise ses racines dans les mêmes nœuds évoqués à propos du manifeste avant-gardiste, dont il représente l'ultime diagnostic de décès et la première métamorphose :

Comment écrire un manifeste d'urbanisme pour la fin $\mathrm{du} \mathrm{xx}^{\mathrm{e}}$ siècle, dans une époque qui a la nausée des manifestes? Les manifestes pèchent fondamentalement par leur manque de preuves. Le problème de Manhattan se pose en termes exactement inverses : c'est une montagne d'évidences sans manifeste. Face à cette double constatation, le présent ouvrage a été conçu comme le manifeste rétroactif de Manhattan. (Koolhaas [1978] 2002 : 9)

28 C'est au cœur de cet inversement du rapport institué par les avant-gardes entre passé et présent, projets et faits, que le geste de Koolhaas se veut un manifeste : rétroactif, son texte prend comme point de départ ces évidences bâties qui faisaient défaut aux manifestes du passé. Prenant appui sur ce qui a été pensé et réalisé par d'autres au cours de l'histoire, le manifeste rétroactif déplace l'épicentre du discours manifestaire : d'un discours d'action nous passons à un discours de réflexion, d'une voix qui se détache du passé pour imposer sa volonté incréée, nous passons à une voix qui se penche sur l'histoire pour en tirer une hypothèse de lecture critique du présent et une projection sur le futur. Il s'agit là d'une nouvelle forme de manifeste qui articule différemment ses composants critique, polémique et programmatique : un manifeste qui se veut un diagnostic à la fois du présent et du futur, par le passé.

Delirious New York se propose ainsi de traduire en manifeste le programme que les architectes de l'âge héroïque du gratte-ciel partageaient de manière plus ou moins consciente, sans pour autant pouvoir l'écrire :

Ce livre est une interprétation de Manhattan qui confère à ses épisodes apparemment discontinus, voir irréconciliables, un certain degré de logique et de cohérence, une interprétation qui entend désigner en Manhattan le produit d'une théorie informulée, le manhattanisme, dont le programme : exister dans un monde totalement fabriqué par l'homme, c'est-à-dire vivre à l'intérieur du fantasme, était d'une ambition telle que pour se réaliser il lui fallait renoncer à toute énonciation explicite. (Koolhaas [1978] $2002: 13$ )

Pour détisser le récit d'un phénomène urbain souvent présenté comme étant le produit spontané d'une exigence d'optimisation, le manifeste de Koolhaas ne se limite toutefois pas aux évidences réelles : son analyse se veut le fruit de l'application à Manhattan de la méthode paranoïa-critique introduite par Salvador Dalí, " délire d'interprétation [qui] consiste à inventer des preuves pour des hypothèses indémontrables et à les greffer dans le monde de la réalité21 $»$ (Koolhaas [1978] $2002: 241$ ).

31 Voici que le réel devient une mine d'évidences disponibles pour que l'on en tire des récits, des manifestes qui n'aspirent plus à l'univocité : comme l'affirme Koolhaas, l'architecture est une "mer" dont, à défaut de pouvoir en résumer la complexité, l'architecte devra surfer sur la vague. Ainsi, le manifeste devient un genre interprétatif 
et la ville se configure comme base réelle d'une narration s'apparentant à un scénario : "Ce livre décrit un Manhattan théorique, un Manhattan comme conjecture, dont la ville actuelle n'est que le compromis" (Koolhaas [1978] 2002 : 11). Dans Delirious New York, Manhattan est lu comme le produit d'une culture de la congestion qui trouve dans la multiplication verticale du site des gratte-ciel la solution apparemment la plus rationnelle pour le maintien tacite de l'irrationalité. Cette culture trouve dans la tour, la grille et la sphère ses coordonnées symboliques ; dans Luna Park de Coney Island son laboratoire formel ; dans la lobotomie entre intérieur et extérieur, conteneur anonyme et contenu hyperspécialisé, la clé de la déprogrammation des destinations fonctionnelles; dans la grille, enfin, le plan d'urbanisme idéal pour héberger des unités constructives conçues comme autant de monades. Si les architectes de l'âge héroïque de Manhattan ont façonné une culture de la congestion, il revient aux architectes de la fin $\mathrm{du} \mathrm{xx}^{\mathrm{e}}$ siècle d'assumer les prétentions de la métropole: ainsi, la conclusionfiction de Delirious New York illustre une série de projets utopiques mâtinés d'humour, qui seraient le produit du manhattanisme amené à ses extrêmes conséquences.

Rem Koolhaas montre une pleine conscience du remaniement définitionnel qu'il est en train d'effectuer sur le genre manifestaire avec l'introduction de sa version rétroactive: la preuve, il n'hésitera pas à doter ce concept d'une généalogie, réabsorbant dans le genre manifestaire Learning from Las Vegas de Robert Venturi. C'est au cours d'une interview en 1999 (Koolhaas 2001 : 593), que Koolhaas fait de cet ouvrage paru en 1972 le dernier manifeste et le premier d'une série de livres sur des villes qui appellent un manifeste, parmi lesquels figure aussi Delirious New York. L'opération conduite ici par Koolhaas est double, car la logique de réécriture de l'histoire du manifeste rétroactif s'applique à la fois à son texte et à celui de Venturi, qui acquiert un statut manifestaire plus de vingt ans après sa rédaction.

Il se trouve que Robert Venturi est aussi l'auteur, en 1966, d'un manifeste titré comme tel: «Nonstraightforward Architecture. A Gentle Manifesto» (Venturi 1966:16) se qualifie dès son titre comme " doux ». Il n'en faut pas davantage pour faire de ce texte l'antithèse d'un manifeste canonique : mis à part le fait que ses formules polies - «I like », «I am for»- n'imposent rien, ce texte prône aussi une complexité contradictoire que le manifeste moderniste excluait a priori. "Une architecture de la complexité et de la contradiction [...] doit embrasser l'unité difficile de l'inclusion plutôt que l'unité facile de l'exclusion. Plus n'est pas moins »: une affirmation qui, renversant le slogan de Mies van der Rohe "moins, c'est plus", sonne comme un anathème, "doucement" lancé contre les racines modernistes de la pensée manifestaire.

Pourtant, pour Rem Koolhaas le véritable manifeste de Venturi est bien ce Learning from Las Vegas qui, à l'instar de Delirious New York, prend comme point de départ les évidences de l'existant pour en tirer un manifeste rétroactif : un manifeste qui puise ses sources dans le passé pour en tirer des hypothèses sur le présent, et par là, des suggestions pour le futur. Pour Koolhaas, le manifeste est ainsi moins une forme discursive et textuelle qu'une fonction conceptuelle reliant de manière spécifique l'architecture avec ses composants programmatique, critique et théorique.

Dans Learning from Las Vegas, l'évidence bâtie s'impose comme le point de départ de toute argumentation qui aspirerait à se tailler un espace de performativité. La ville y est interprétée comme un manifeste vivant, un conteneur d'inventions que l'architecte est appelé à s'approprier : le catalogage de Las Vegas est la condition de possibilité pour 
l'élaboration rétroactive du manifeste tacite de toute une génération d'architectes qui ont dénaturé les prémisses du modernisme, en en faisant une forme non avouée d'expressionnisme de la structure. Pour Venturi cette dérive expressionniste - incarnée par la figure du bâtiment « canard »- était déjà implicite dans la contradiction entre les deux impératifs du modernisme, l'élimination de la décoration et le triomphe de la fonction: là où la décoration ne peut véhiculer le message du bâtiment, c'est la structure qui se plie à des finalités symboliques. À l'opposé du canard, le " hangar décoré » de Las Vegas est un phénomène de communication architecturale adapté à la vitesse de la circulation en voiture. Il s'agit là d'une architecture fonctionnelle, associant un bâtiment anonyme, modulaire et pas cher à une enseigne ou strip ; à savoir une couche symbolique évolutive qui se fait charge des exigences signalétiques de tout bâtiment commercial. Voici qu'avec les strips, «le signe graphique dans l'espace devient l'architecture du paysage » : à Las Vegas, sur la Route 91, « si on enlève les enseignes, il n'y a pas de lieu ».

En dépit des différences d'approche qui caractérisent les deux ouvrages, le parti pris conceptuel de Learning from Las Vegas est le même qui guidait Delirious New York: à partir de ce qui existe, Venturi dresse une archéologie qui relie les sources du passé et les formes du présent dans un discours conçu pour ouvrir des pistes pour le futur. Venturi se propose en cela d'inverser le rapport «intolérant" (Venturi, Brown et Izenour [1972] 2008: 17) que le modernisme avait institué avec le réel: tirer un manifeste du programme implicite dans une série d'évidences bâties, implique d'accepter le réel comme source d'enseignement. Le déchiffrement du présent devient alors un projet à part entière, voire une activité d'avant-garde. La racine polémique, et finalement le caractère manifestaire du livre de Venturi, résident ainsi dans une prise en compte de la productivité du symbolisme du laid et de l'ordinaire des architectures de Las Vegas :

Étudier le paysage existant est pour un architecte une manière d'être révolutionnaire [...] pas à la manière trop évidente qui consisterait à détruire Paris et à le recommencer comme Le Corbusier le suggérait vers 1920, mais d'une manière plus tolérante : celle qui questionne notre façon de regarder ce qui nous entoure. (Venturi, Brown et Izenour [1972] 2008 : 17)

L'approche postmoderne concrète et redevable des intérêts de l'histoire de Venturi se dresse dès lors contre le mythe de l'originalité du modernisme. C'est là aussi la racine des polémiques que l'ouvrage de Venturi a suscitées : car, selon les points de vue, entre le monde tel qu'il est et tel qu'il devrait être, se dessine la frontière entre une acceptation productive du réel et un adieu à la force critique de l'utopie.

Ainsi, Delirious New York et Learning from Las Vegas ont marqué un tournant dans le rapport que l'architecture entretient avec sa théorie, mais ils ont ouvert aussi un horizon de développement inédit pour le manifeste en architecture : voici que, à partir de sa version rétroactive, le manifeste architectural ne cessera plus d'évoluer, jusqu'à esquisser les contours d'un retour du manifeste au XXI ${ }^{\mathrm{e}}$ siècle.

\section{Le manifeste contemporain : collectivement singuliers}

Les cas de Conrads, de Venturi et de Koolhaas ont montré le rapport étroit qui lie les critères de sélection et de labellisation du manifeste avec le type d'histoire que ce dernier permet d'écrire. Le panorama contemporain fait de ce même nœud un dilemme 
radical: c'est d'un choix définitionnel que dépendront en très grande partie les conclusions que l'on pourra tirer sur le destin du manifeste après sa «mort ». Ainsi le deuxième graphique que nous avons examiné sera-t-il valable seulement à condition d'inclure dans une généalogie du genre deux ouvrages qui mettent en échec toute définition classique du manifeste. Ces textes ont en effet été écrits en réponse à une commande et en vue d'une publication dans des recueils collectifs : il n'en faut pas plus pour mettre en crise la prétendue spontanéité du manifeste avant-gardiste. Si déjà en 1926 les éditeurs de la revue $G^{22}$ incitaient leurs lecteurs à écrire des manifestes, le recueil de manifestes sur commande est, à notre connaissance, une forme contemporaine.

Le premier recueil pris en compte dans le graphique a été publié en août 2007 pour célébrer le cinquantième numéro de la revue d'architecture Icon: le dossier «Fifty manifestos " rassemble cinquante manifestes, tous intitulés comme tels et numérotés, signés par des architectes, des designers, des critiques. Si ce dossier s'ouvre sous le signe d'un constat négatif classique - «L'âge du manifeste est fini. Les grandes idéologies sont mortes»-, il préconise aussi de nouvelles perspectives pour le manifeste contemporain, à condition, toutefois, que ce dernier accepte de changer du point de vue des formes, des finalités et des canaux - en un mot, d'identité :

Mais quoi à propos d'aujourd'hui ? De nos jours [...] n'importe qui peut publier sa lamentation sur internet. Le manifeste est de retour! [...] Au début du xxi siècle, il y a tant de manifestes potentiels qu'il y a de personnes - internet le rend possible. (McGuirk 2007 : 36)

L'analyse d'une page du dossier suffit en effet pour comprendre que, si le manifeste est de retour, il n'a plus grand-chose en commun avec ses antécédents avant-gardistes, mis à part un titre qui pourrait s'avérer une coquille vide. Si Marinetti scandait le lancement des tracts futuristes, affirmant que «les manifestes, en se cumulant, se détruisent entre $\operatorname{eux}^{23}$ ", les manifestes d'Icon cohabitent sur une même page et partagent les mêmes choix de mise en page. Qui plus est, parmi ces textes très peu prennent position: la plupart se bornent à tisser un jeu de renvois formels et de détournements vis-à-vis de l'imaginaire associé au manifeste moderniste. À son tour, l'ordre de publication des textes n'est pas sans implications: si le Manifesto \#01, signé par Peter Saville ( Be careful of what you wish»), place le recueil sous le signe du doute, le Manifesto \#50 de MUF Architects fait des manifestes d'antan le rappel des vernissages mondains d'aujourd'hui : "Vous êtes cordialement conviés au lancement du manifeste MUF [...] un buffet sera servi. »

Ces manifestes qui ne craignent pas de se confondre sur une même page, se laissent aussi archiver par thèmes, renversant le caractère individualiste typique des manifestes collectifs des avant-gardes. Ainsi, à la fin du magazine, une liste présente le palmarès des concepts les plus récurrents dans les manifestes, dressant un portrait du cadre de valeurs de l'architecture contemporaine : d'« innovation » (12 occurrences), à " moralité », grand dernier avec un seul résultat.

Du point de vue des formes et des canaux de diffusion, il est intéressant de remarquer que, si le dossier a été tiré à plusieurs milliers de copies, chaque texte a aussi été publié sur le site de la revue: les versions numériques de ces manifestes ont totalisé entre 6000 et 12000 vues. Ces chiffres dépassent les tirages souvent restreints des manifestes avant-gardistes : en filigrane se dessine alors la question des critères de mesure de l'espace de performativité supposé ou réel des manifestes au fil du temps. Il est 
toutefois difficile d'estimer l'impact réel de ces décomptes, compte tenu de nouvelles formes de lecture sur Internet, s'apparentant à un balayage rapide d'informations. Ainsi, la diffusion du manifeste sur Internet demande le développement d'outils d'analyse capables de faire face d'une part à une disponibilité presque illimitée de données opaques, de l'autre à des pratiques discursives qui redessinent le visage du manifeste. Comme l'affirme Beatriz Colomina: "Nouveaux médias = Nouveaux manifestes (mais ils pourraient ne plus ressembler à des manifestes)» (Colomina 2014 : n. p.).

Publié en 2010 par le MAK Center de Los Angeles, le deuxième recueil inclus dans le graphique se veut l'expression d'un propos moins événementiel que le dossier d'Icon: les trente-deux manifestes de Urban Future Manifestos (Noever et Meyer 2010) sont le fruit d'une série d'invitations et d'un programme de résidences lancés à partir de 2008 dans le cadre de la Urban Future Initiative ${ }^{24}$. Parmi les contributeurs, les boursiers du programme UFI, des critiques et des architectes (Michael Sorkin, Lebbeus Woods), mais aussi des artistes célèbres (James Turrell, Ai Weiwei).

Sur la quatrième de couverture, les éditeurs du recueil Peter Noever et Kimberli Meyer résument ainsi l'esprit du projet :

Urban Future Manifestos se propose de servir à ses lecteurs en présentant une collection de positions intellectuelles et imaginatives au format poche. Une constellation de points de vue émerge de ces textes, représentant les réverbérations des idées en jeu dans les sphères critiques et créatives de nos jours.

Cette idée d'une constellation de points de vue pourrait suggérer la transition du manifeste, de texte collectif relevant de la polémique et d'une théorie militair ${ }^{25} \mathrm{du}$ pouvoir symbolique, à geste de prise de parole individuelle dans une arène publique : la diffusion du manifeste au singulier est en effet l'un des traits caractéristiques des évolutions récentes de cette pratique discursive dans tous les domaines de la création (Birolli et Tjell 2013 : 7-14).

S'agirait-il du retour d'une définition ancienne du manifeste, conçu comme proclamation individuelle d'intentions ${ }^{26}$, dans un cadre socio-discursif contemporain faisant de la galaxie de points de vue une nouvelle force critique, et des cadres institutionnels indépendants (laboratoires, centres d'art, project space) autant d'espaces pour penser - mais comment?- le renouveau? Cette émergence de «formes manifestaires par cumulation » pourrait dès lors être interprétée comme la réponse productive à un manifeste ayant perdu sa force de frappe ; mais elle pourrait aussi être lue comme le signe du passage du manifeste de geste de rupture à simple exercice de style. Ainsi, ces recueils manifestaires s'avèrent souvent des objets malaisés à traiter : à défaut d'une opinion forte qui se dégagerait entre les lignes des textes qui les composent ${ }^{27}$, le critique est souvent obligé de s'en tenir soit à une analyse parcellaire de chaque texte, soit à un examen du cadre qui prescrit a priori l'intention, encore plus que la nature manifestaire des textes et du recueil.

Dans l'introduction de Urban Future Manifestos, cette intention manifestaire se nourrit de l'élan d'un texte de George Orwell, dont Peter Noever choisit de dédoubler la dystopie - de 1984 à Metropolis 2084 (ou George Orwell revisited : 1984) :

La ville est l'habitat du présent et le biotope du futur [...]

L'utopie n'est plus un idéal

L'utopie est le potentiel du réel à transformer [...]. 
Vis-à-vis de cette prise de position utopique d'il y a un demi-siècle, l'auteur contemporain ne peut que prendre une position nuancée: d'une part, il en constate l'échec, d'autre part, il choisit d'en relancer l'actualité dans la forme d'un appel - «le défi de notre présent est de faire face aux questions urgentes de l'urbanisme, car c'est ça qui va décider de la forme que prendra notre futur». Voici que la volonté de relancer le manifeste prend parfois la forme d'un défi lancé contre ses propres hésitations : manifeste malgré tout. Voici que le manifeste en architecture est, encore une fois, une manière pour questionner le rapport entre la ville telle qu'elle est et telle qu'elle pourrait être, en théorie et en pratique.

Qu'ils soient faibles ou hybrides, cyniques ou nostalgiques, ces recueils manifestaires ne sont pas des cas isolés : ils s'inscrivent dans le cadre d'un retour d'intérêt plus ample pour le manifeste en architecture, marqué par des formats discursifs, éditoriaux et événementiels encore à explorer.

\section{Conclusion : quel futur pour le manifeste?}

51 Le panorama des manifestes en architecture de la première décennie du xxi siècle se présente comme un terrain d'étude flou; un regard sur la production manifestaire récente témoigne toutefois moins d'un manque de manifestes que de catégories critiques capables d'encadrer des formes contrevenant aux définitions classiques du genre.

52 L'une des formes de ce retour métamorphosé du manifeste, que nous avons essayé de mettre en perspective avec le tournant réflexif imprimé au genre par Rem Koolhaas, consiste en la récupération du manifeste de la part des théoriciens, en tant qu'outil critique d'écriture de l'histoire. Nous citerons à titre d'exemple Theories and Manifestoes of Contemporary Architecture de Charles Jencks, de 1997; Chronomanifestes 1950-2010 de Bernard Tschumi, de 2013; et Manifesto Architecture : The Ghost of Mies de Beatriz Colomina, de 2014. Trois ouvrages qui, de manière différente, redéfinissent et façonnent le manifeste en fonction du regard que chacun des auteurs porte sur l'histoire du genre et de l'architecture; trois textes où la volonté manifestaire semblerait parfois appartenir plus aux historiographes qu'à l'histoire.

Charles Jencks définit le manifeste comme texte à mi-chemin entre un volcan, explosif, et une tablette de lois, prescriptive ; c'est à partir de cette notion du manifeste comme " format ", que l'auteur procède à la construction des textes de son recueil, obtenus en recoupant des passages «manifestaires » dans des textes plus longs. Le moins que l'on puisse dire de ce panorama des manifestes en architecture entre 1954 et 2005, est qu'il s'agit d'une interprétation artificielle du sujet: parmi les textes du recueil, deux seulement portent dans leur titre le mot « manifeste».

54 Pour Bernard Tschumi, tout au contraire, le manifeste est moins un format qu'une question de contenu et d'interprétation. Ainsi, le recueil qu'il édite en 2013 rassemble autour de la notion d'« idée-manifeste » une série de projets qui, entre 1950 et 2010, ont marqué l'histoire de la discipline, selon une approche où le caractère "manifestaire " de chaque projet et les péripéties de sa réception se confondent : "Avant d'être des formes, c'est d'idées qu'il s'agit » (Tschumi 2013 : 9).

Expression d'une conception de l'architecture en tant que phénomène culturel complexe, l'intérêt de Tschumi pour le manifeste remonte à 1978, lors de son 
exposition Architectural Manifestos à l'Artist Space de New York. Encore une fois, le manifeste était ici une manière de questionner et mettre en mouvement le rapport et la hiérarchie entre l'espace architectural et sa conceptualisation théorique, forme bâtie, projet et idée : "Je n'avais pas à bâtir - les livres étaient architecture, les expositions étaient architecture, et les publicités étaient architecture » (Buckley et Craig 2015 : 178) affirmera-t-il des années plus tard à propos de cette période de sa recherche. Le manifeste sur le manifeste qui introduit l'essai de Beatriz Colomina, quant à lui, redéfinit le manifeste à partir d'un autre aspect de son fonctionnement, à savoir son rapport avec les médias : «1. Le manifeste est un média. Il n'existe pas en dehors d'un autre média (journaux, revues, pamphlets, affiches, radio, etc.) »(Colomina 2014 : n. p.). Le constat du lien, chez les avant-gardes historiques, entre l'avènement du manifeste et un intérêt accru pour les médias permet à l'auteure d'inclure dans son analyse du genre tous ces textes polémiques et ces revues programmatiques qu'ont contribué à faire de l'œuvre d'architectes modernistes comme Adolf Loos et Mies van der Rohe des manifestes à part entière. C'est cette définition large de l'objet manifestaire qui permet aussi à Colomina de réabsorber dans le genre des pratiques extratextuelles, comme les pavillons éphémères des expositions universelles et, plus récemment, ceux de la Serpentine Gallery.

Dans la généalogie qui en résulte, les manifestes bâtis ou dessinés du passé trouvent un écho dans les manifestes contemporains : du Pavillon allemand de Mies van der Rohe pour l'Exposition internationale de Barcelone (1929), à l'installation "soft manifesto " présentée par SANAA (Kazuyo Sejima et Ryue Nishizawa) en 2008 dans ce même pavillon, en passant par la Casa Palestra conçue par l'agence OMA pour la $17^{\mathrm{e}}$ Triennale de 1986. "4. Chaque manifeste retravaille les manifestes précédents. L'appel au nouveau se construit sur l'ancien» (Colomina 2014 : n. p.) : et si la proposition de Rem Koolhaas avait été prophétique, et le manifeste de nos jours était destiné à être le fruit d'un regard rétroactif?

S'il y a un aspect commun à ces reprises du manifeste, il s'agit en effet de leur rôle dans l'articulation et l'évolution des rapports que les architectes du passé ou du présent instituent entre représentation et réalisation architecturale, architecture comme discipline pratique et comme démarche théorique.

Qu'il prenne la forme d'une reprise de l'esprit manifestaire d'antan ou d'un intérêt théorique renouvelé de la part des institutions et de la critique, ce retour récent du manifeste se prête à des évaluations différentes, entre métamorphose productive, réification neutralisante et effet de mode, voire recyclage nostalgique d'un mythe ${ }^{28}$ avant-gardiste. Plus intéressant est que cet intérêt pour une histoire lue au prisme du manifeste s'accompagne d'un retour, pendant la première décennie du $\mathrm{XxI}^{\mathrm{e}}$ siècle, de manifestes écrits par des architectes.

Loin de porter un jugement sur leur valeur, nous nous limiterons à souligner l'éventail de formats dont font preuve les manifestes architecturaux récents : de la transcription d'interventions orales (Koolhaas 2014) à des manifestes rédigés à l'occasion d'une exposition (Nouvel 2005) ou d'un événement tel le Serpentine Gallery Manifesto Marathon (Obrist et Peyton-Jones 2008); d'ouvrages manifestaires dépourvus de charge polémique (Geddes 2013) à des textes qui sembleraient vouloir réactualiser l'esprit tapageur des manifestes d'avant-garde (Betsky et Adigard 2000). La question d'une reconsidération de la définition et des visées du manifeste se pose face à ces nouveaux textes individuels qui se revendiquent comme des manifestes et qui sembleraient 
vouloir en renouveler l'esprit, au-delà d'un simple renvoi nostalgique à l'avant-garde : "Le temps scintille et le songe est savoir », comme l'écrit Jean Nouvel (2005) en citant Paul Valéry.

61 Hybrides, détournées, marginales, réactivées ou lancées avec un enthousiasme renouvelé, ces nouvelles formes pourraient dès lors être considérées comme les figures d'un âge du manifeste "après le manifeste ", dont l'histoire serait en train de s'écrire. La question de l'inscription d'un nouveau chapitre dans l'histoire du manifeste, conçu moins comme genre que comme pratique adaptative, n'est pas, de notre point de vue, à relier de manière exclusive avec la valeur et l'efficacité que l'on voudra reconnaître à ces formes.

Loin de vouloir aboutir à des conclusions univoques, au fil de cette histoire nous avons essayé de montrer que la construction de l'histoire de l'architecture à travers le prisme des manifestes et la construction du manifeste en tant qu'objet théorique croisent souvent leurs chemins; que les rapports entre pratique et définition du manifeste ne cessent d'évoluer; que les liens entre manifeste et effet manifestaire engendrent un jeu d'échos qui permet de complexifier la linéarité du récit par ruptures successives façonné par le manifeste : "Le manifeste ne se borne pas à décrire une histoire de la rupture, il produit une histoire de ce type » (Puchner 2002 : 451).

63 Les opacités interprétatives du manifeste de Sant'Elia, les oscillations entre manifeste et programme chez Conrads, le manifeste rétroactif de Koolhaas et les essais récents de redéfinition du genre peuvent dès lors être lus comme autant d'étapes d'une histoire à la fois du manifeste et de l'architecture : histoire des rapports vastes et changeants entre projet et idée, héritage d'hier, pratique du présent et vision du lendemain.

\section{BIBLIOGRAPHIE}

Abastado, Claude et Gleize, Jean-Marie et al., 1980, Littérature, $\mathrm{n}^{\circ} 39$.

Banham, Reyner, 1957, « Poetica di Sant'Elia e ideologia futurista », Architettura. Cronache e storia, a. II, $\mathrm{n}^{\circ} 15$, p. 626.

Betsky, Aaron, Adigard, Erik, 2000, Architecture Must Burn. A Manifesto for an Architecture Beyond Building, Londres, Thames \& Hudson.

Birolli, Viviana, 2008, Manifesti del futurismo, Milan, Abscondita.

Birolli, Viviana et Bloomfield, Camille et al., 2013, « Manart : une base de données sur les manifestes artistiques et littéraires au xx ${ }^{\mathrm{e}}$ siècle », Études Littéraires, vol. 44, nº 3, p. 149-150.

Birolli, Viviana et Tjell, Mette (dir.), 2013, Études Littéraires, vol. 44, n 3, « Manifeste/s ».

Bloomfield, Camille et Tjell, Mette, 2013, « Les âges d'or du manifeste artistique et littéraire en France : étude contrastive à partir de la base de données Manart ", Études littéraires, vol. 44, $\mathrm{n}^{\circ} 3$, p. 151-163. 
Boschetti, Anna, 2010, « La notion de manifeste », Francofonia, $n^{\circ}$ 59, « Les manifestes littéraires au tournant du XXI ${ }^{\mathrm{e}}$ siècle », p. 13-29.

Buckley, Craig, 2015, After the Manifesto, actes du colloque « What Happened to the Architectural Manifesto? », GSAPP, Columbia University, 18 novembre 2011, New York, Columbia GSAPP Books on Architecture, Columbia Press.

Carrà, Carlo Dalmazzo, 1981, La mia vita, Milan, Feltrinelli.

Caruso, Luciano, 1980, Manifesti, proclami, interventi, e documenti teorici del futurismo, 1909-1944, Florence, SPES-Salimbeni.

Colomina, Beatriz, 2014, Manifesto Architecture: The Ghost of Mies, Berlin, Sternberg Press. Conrads, Ulrich, [1964] 1996, Programme und Manifeste zur Architektur des 20, Berlin, Ullstein, Cambridge, MIT Press, 1970, Paris, Éditions de la Villette.

Drudi Gambillo, Maria et Fiori, Teresa, 1958, Archivi del futurismo, v. I, Rome, De Luca. Dumasy, Lise et Massol, Chantal, 2001, Pamphlet - Utopie - Manifeste XIX et XX siècles, Paris, L'Harmattan.

Furetière, Antoine, 1727, Dictionnaire universel de la langue française, t. III, La Haye. Geddes, Robert, 2013, Fit. An architect's manifesto, Princeton, Princeton University Press. Gleize, Jean-Marie, 1980, « Manifestes, préfaces. Sur quelques aspects du prescriptif », Littérature, $\mathrm{n}^{\circ} 39$, p. 12-16.

Gnocchi, Maria Chiara, 2010, «Lire les manifestes littéraires à l'orée du xxI ${ }^{\mathrm{e}}$ siècle », Francofonia, $\mathrm{n}^{\circ} 59$, p. 5-11.

Icon, Manifesto Issue, $\mathrm{n}^{\circ}$ 50, août 2007, www.iconeye.com, consulté le 21 mars 2017.

Jaudon, Raphaël et Birolli, Viviana et al., 2015, « Manifestes », Marges, $\mathrm{n}^{\circ} 21$.

Jencks, Charles et Kropf, Karl, 1997, Theories and Manifestoes of Contemporary Architecture, Chichester, Academy Editions.

Kaplan, Alice, 1983, « Recent Theoretical Work with Pamplets and Manifestoes », L'Esprit créateur, vol. 23, n 4, p. 74-82.

Koolhaas, Rem et al., 2001, Harvard Design School Guide to Shopping (Project on the City), vol. 2, Cologne, Taschen.

Koolhaas, Rem, [1978] 2002, Delirious New York. A Retroactive Manifesto for Manhattan, New York, Monacelli Press, 1978, New York délire, Marseille, Éditions Parenthèses.

Koolhaas, Rem, 2014, Preservation is overtaking us. Supplement to OMA's Preservation Manifesto, by Jorge Otero-Pailos, Columbia, GSAPP Books, « Relearning from Las Vegas: Then and Now », interview de Hans Ulrich Obrist et de Koolhaas à Robert Venturi et à Scott Brown, Genève, 1999. Larue, Anne (dir.), 2008, Itinéraires et contacts de cultures, n 43, « L'art qui manifeste ». Leroy, Claude, 1980, « La fabrique du lecteur dans les manifestes », Littérature, nº 39, p. 120-128. Lista, Giovanni, 1973, Futurisme. Manifestes - documents - proclames, Lausanne, L'Âge d'Homme. Loos, Adolf, 1910, « Ornament und Verbrechen » [Ornement et crime], Der Sturm, Berlin, avril 1910. Mann, Paul, 1991, The Theory-Death of the Avant-Garde, Bloomington, Indiana University Press. Margel, Serge, 2013, « Le temps du manifeste », Lignes, $\mathrm{n}^{\circ}$ 40, p. 5-7. 
Mcguirk, Justin, 2007, « Icon of the Month. The Manifesto », Icon, p. 36.

Meizoz, Jérôme, 2007, Postures littéraires. Mises en scène modernes de l'auteur, Genève, Slatkine.

Mertins, Detlef et Jennings, Michael W., 2010, G: An Avant-Garde Journal of Art, Architecture, Design, and Film, 1923-1926, Los Angeles, Getty Research Institute.

Millot, Hélène, 2001, «Vertus du nombre : les procédés cumulatifs dans la pratique manifestaire (1886-1920) », dans L. Dumasy et C. Massol (dir.), Pamphlet - Utopie - Manifeste XIX et XX ${ }^{e}$ siècles,

Paris, L'Harmattan, p. 223-235.

Noever, Peter et Meyer, Kimberli, 2010, Urban Future Manifestos, Los Angeles, MAK Center, Hatje Cantz.

Nouvel, Jean, 2005, Louisiana Manifest, catalogue de l'exposition, Louisiana Museum, du 7 juin au 18 septembre 2005, Humlebæk, Louisiana.

Obrist, Hans Ulrich et Peyton-Jones, Julia (dir.), 2008, Serpentine Gallery Manifesto Marathon, Serpentine Gallery, Londres, Koenig Books.

Puchner, Martin, 2002, Theatre Journal, vol. 54, $\mathrm{n}^{\circ}$ 3, « Manifesto = Theatre ».

Schaeffer, Jean-Marie, 1989, Qu'est-ce qu'un genre littéraire, Paris, Seuil.

Southern, John, 2011, « Urban Future Manifestos », Domus, Milan, novembre 2011, [En ligne], https://www.domusweb.it/en/reviews/2011/11/25/urban-future-manifestos.html, consulté le 15 septembre 2018.

Tschumi, Bernard, 1978, Drawing and Installation by Bernard Tschumi. Architectural Manifestos, catalogue de l'exposition, Artists Space, du 8 au 29 avril 1978, New York.

Tschumi, Bernard, 2013, Chronomanifestes 1950-2010, catalogue de l'exposition, Les Abattoirs, Toulouse, du 28 septembre 2013 au 5 janvier 2014, Orléans, Éditions HYX, coll. « Frac Centre ».

Venturi, Robert, 1966, Complexity and Contradiction in Architecture, New York, MoMA.

Venturi, Robert, Brown, Scott et Izenour, Steven, [1972] 2008, L'enseignement de Las Vegas, Bruxelles, Mardaga.

\section{NOTES}

1. "L'époque des manifestes est close» (Gleize 1980: 13). "On pourrait croire que l'art qui manifeste est une vieille lune » (Larue 2008 : 7). Dans la critique des années 1980, cette idée d'une «mort» du manifeste est souvent solidaire (voire partiellement instrumentale) de la fin de la période des avant-gardes dites « historiques».

2. «Les manifestes des années 1970 se présentent comme des jeux intellectuels [...] par là même, ils semblent indiquer la remise en cause, sinon le déclin, du genre. Mais la mort des manifestes, imminente et définitive aux yeux de plus d'un, n'a pas eu lieu » (Gnocchi $2010: 6$ ).

3. «Le manifeste est un genre littéraire qui se confronte à la monté des avant-gardes, mais ne s'y réduit pas» (Margel $2013: 6$ ).

4. Entre autres, Enrico Prampolini, «L'atmosferastruttura. Basi per un'architettura futurista ", publié dans Il Piccolo Giornale d'Italia, a. III, nº 29, 29-30 janvier 1914 et dans Noi, a. II, $n^{\circ}$ 2-3-4, Rome, février 1918; Theo Van Doesburg et al., "Manifest I van De Stijl », De Stijl, a. II, nº 1 , novembre 1918, p. 2; Naum Gabo et Antoine Pevsner, "Manifeste Réaliste ", tract lancé à Moscou, 5 août 1920 ; Theo Van Doesburg, « Manifeste V », De Stijl, 1923, texte publié à l'occasion de l'exposition De Stijl à la galerie L'Effort Moderne de Paris; Oskar Schlmmer, « Manifesto for 
the first Bauhaus exhibition ", tract publié à l'occasion de la première exposition du Bauhaus à Weimar, juillet-septembre 1923.

5. Entre autres, Marinetti, Filippo Tommaso, Mazzoni, Angiolo, Somenzi, Mino, «Manifesto futurista dell'architettura aerea », Sant'Elia/Futurismo, a. II, $\mathrm{n}^{\circ} 3,1^{\mathrm{er}}$ février 1934.

6. Entre autres, Alison Smithson et Team X, «Statement on Habitat. The Doorn Manifesto », tract lancé en 1954; Friedensreich Hundertwasser, «Verschimmelungs-manifest gegen den rationalismus in der architektur ", manifeste lu dans l'abbaye de Seckau en 1958, puis présenté pendant une conférence à la Concordia Hall, Vienne, le 9 février 1968 ; Reinhard Gieselmann et Oswald Mathias Ungers, «Towards a New Architecture », publié dans Der Monat, n 174, Berlin, mars 1963.

7. L'étude conduite par Mette Tjell et Camille Bloomfield (2013) sur les manifestes artistiques produits en France entre 1886 et 2009 semblerait confirmer ce retour du manifeste à partir des années 2000.

8. «De plus, dans les processus historiques, la chose est souvent indépendante du mot, et la perception est un facteur essentiel dans la genèse des classements des phénomènes intellectuels et sociaux » (Boschetti $2010: 13$ ).

9. En 1980, Claude Abastado soulignait déjà le poids de la réception dans la construction du phénomène manifestaire. "Les distinctions entre manifeste, proclamation, appel, adresse, préface, déclaration sont fragiles; les circonstances historiques et la réception des textes, la manière dont ils sont entendus, lus, interprétés, entraînent des glissements de qualifications [...]. Par comparaison - et par anachronisme - on appelle "manifestes" tous les textes programmatiques et polémiques, quelles qu'en soient les formes. Celles-ci dépendent de la forme de la communication: les techniques historiquement évoluent, les institutions de diffusion se transforment, le public change » (Abastado $1980: 4$ ).

10. Sant'Elia, Antonio, «L'architettura futurista. Manifesto » (Birolli 2008: 138-142) ; «L'architecture futuriste - Manifeste » (Lista $1973: 233-235)$.

11. Le manifeste est publié dans Lacerba, $n^{\circ} 15$, Florence, $1^{\text {er }}$ août 1914 ; des extraits en français sont ensuite publiés dans NOI, II série, $\mathrm{n}^{\circ}$ 10-11-12, Rome, 1925.

12. Le manifeste «Ricostruzione futurista dell'universo» est signé par Giacomo Balla et Fortunato Depero et diffusé comme tract de la Direction du mouvement futuriste avec la date 11 mars 1915.

13. Prima Esposizione d'Arte del Gruppo Nuove Tendenze, catalogue de l'exposition, Famiglia Artistica, Milan, 20 mai-10 juin 1914.

14. Entre autres, Carlo Accetti, "Sant'Elia fu o no futurista?", La Provincia di Como, Côme, 19 octobre 1956, p. 3 ; Bruno Zevi, "Sant'Elia non era futurista », L'Espresso, Rome, 2 septembre 1956, p. 15 ; Aldo Carpi de' Resmini, « Sant'Elia non era futurista », L'Architettura, décembre 1956, p. 549.

15. Pour une confrontation entre les deux textes voir Casabella, VII, $\mathrm{n}^{\circ} 82$, octobre 1934, p. 516-517. Parmi les ajouts de Marinetti, nous mentionnons toute la première partie polémique (de « Après le XVIII ${ }^{\mathrm{e}}$ siècle il n'y a plus eu aucune architecture [...] » à " [...] la maison et la ville spirituellement et matériellement nôtres, dans lesquelles notre tumulte puisse s'exprimer sans paraître un gigantesque anachronisme ») ; l'attaque aux architectures étrangères (point n. 1 de la liste «Je combats et je méprise »); l'idée du caractère éphémère de l'architecture (« Les maisons durerons moins que nous ", point $n^{\circ} 8$ de la liste « Je proclame »).

16. Voir C. M. Boesmi, «Commemorazione veloce di Sant'Elia», Regime fascista, Cremone, 21 septembre 1930; Filippo Tommaso Marinetti, «Le onoranze dell'architetto Antonio Sant'Elia ", Il Giornale dell'arte, Milan, 21 septembre 1930 ; voir aussi la revue Sant'Elia, dirigée par Mario Somenzi et Angiolo Mazzoni dès octobre 1933, et Filippo Tommaso Marinetti, Ricostruire l'Italia con architetture futuriste Sant'Elia, «divertissement représentable en de nombreuses synthèses ", 1930. 
17. «Le bon lecteur du manifeste ne lit pas : il effectue la demande du texte, il exécute l'effet dont il est la cible, il adhère aux valeurs qui lui sont administrées, mais ne lit pas » (Leroy 1980 : 124).

18. Or mention d'une édition française en bibliographie, les traductions des citations vers le français sont de l'auteur.

19. Signé par Le Corbusier et Pierre Jeanneret, ce texte est publié dans la revue L'Architecture vivante $\left(\mathrm{n}^{\circ} 17,1927\right)$ et dans Alfred Roth, Zwei Wohnhäuser von Le Corbusier und Pierre Jeanneret, Stuttgart, Akadem. Verlag Dr. Fr. Wedekind \& Co., 1927.

20. Si la plupart des chercheurs s'accordent pour reconnaître une stabilité de traits formels et fonctionnels au manifeste d'avant-garde, l'opportunité de considérer le manifeste comme un genre ne cesse de susciter le débat. "À l'intersection du littéraire, de l'historique, de l'idéologique et du stratégique, le manifeste littéraire apparaît comme un objet particulièrement malaisé à définir et à identifier : bien que sa qualité de manifeste semble relever de l'évidence intuitive et/ou empirique, il est difficile d'établir selon quels critères un texte peut ou non être qualifié de manifeste, et bien qu'il présente des constantes formelles, discursives et fonctionnelles, on ne peut pas dire pour autant qu'il constitue un genre littéraire » (Millot 2001 : 222).

21. «L'architecture elle-même est d'autre part inévitablement une forme d'activité PC: "Architecture = imposer au monde des structures qu'il n'a jamais réclamées et qui n'existaient jusque-là que comme projets nébuleux dans le cerveau de leurs créateurs". Cette transition entre une hypothèse spéculative et un être-là irrécusable constitua un véritable traumatisme pour l'architecture moderne » (Koolhaas [1978] 2002 : 246).

22. «Mettez au pilon vos manuscrits! Écrivez des manifestes pour nous! Vivez pour les choses qui existent aujourd'hui »; "History Is What Is Happening Today », G: Material zur elementaren Gestaltung, $\mathrm{n}^{\circ}$ 5-6, avril 1926 (Mertins et Jennings $2010: 229$ ).

23. «Les manifestes, [comme les nouvelles sur la presse] en se cumulant, se détruisent entre eux » (Drudi Gambillo et Fiori 1958 : 295).

24. www.makcenterufi.org. Le programme est né pour favoriser les échanges internationaux et le développement de conceptions urbaines pour le futur.

25. «Certes, le manifeste n'est pas d'abord un genre, mais un geste, un acte: il relève prioritairement d'une pragmatique du discours [...] d'une théorie militaire $\mathrm{du}$ pouvoir symbolique » (Gleize $1980: 12$ ).

26. «Manifeste, s.m. : Est une déclaration que font les Princes par un écrit public des intentions qu'ils ont en commençant quelques guerres, ou autres entreprises, et qui contient les raisons et moyens sur lesquels ils fondent leur droit et leurs prétentions » (Furetière 1727).

27. «Ce qui fait défaut au livre, c'est une position définitive quant à l'avenir de l'urbanisme. Comme c'est le cas de beaucoup de spéculations utopiques, [...] le futur de la métropole mondiale semble dépendre de qualités qui sont des idées génériques [...]. Bien que divertissant à lire, les manifestes de critiques établis ne proposent pas de solutions concrètes » (Southern 2011).

28. «Le discours de la mort de l'avant-garde est aussi le discours de sa récupération » (Mann 1991 : 32). 


\section{RÉSUMÉS}

L'article explore quelques-unes des étapes marquantes de l'histoire du manifeste en architecture, du manifeste futuriste d'Antonio Sant'Elia (1914) aux recueils manifestaires sur commande de la première décennie du XxI ${ }^{\mathrm{e}}$ siècle, en passant par le manifeste rétroactif de Rem Koolhaas (1978). Une confrontation entre deux graphiques issus de la base de données des manifestes Manart est le point de départ pour questionner les difficultés définitionnelles posées par les évolutions les plus récentes des formes et des fonctions du manifeste. Ainsi l'histoire des manifestes est-elle mise en miroir avec une histoire des évolutions de la notion de manifeste, au carrefour entre dynamiques de production textuelle et de réception critique. En conclusion, le constat de la mort du manifeste formulé à partir des années 1980 est problématisé à la lumière du récent retour d'intérêt pour le manifeste en architecture, dans des formes et via des canaux encore en grande partie à explorer.

The article explores some of the milestones in the history of the architectural manifesto: from Antonio Sant'Elia's Futurist manifesto (1914) to the anthologies of the first decade of the twentyfirst century, through Rem Koolhaas' retroactive manifesto (1978). Two graphs from the Manart database are the starting point for questioning the manifestos' definitional difficulties, especially regarding its recent developments in terms of form and function. The links between the history of manifestos and the evolution of its notion are then outlined, a dynamic situated at the crossroads between textual production and critical reception. Following the renewed interest for the manifesto in architecture-the forms of which are yet to be explored-this paper puts into perspective the idea, introduced by the 1980s, that the manifesto has become an obsolete format.

\section{INDEX}

Keywords : manifesto, architecture, Manart, retroaction, hybridization, futurism, Rem Koolhas Mots-clés : manifeste, architecture, Manart, rétroaction, hybridation, futurisme, Rem Koolhas

\section{AUTEUR}

\section{VIVIANA BIROLLI}

Université Paris 1 Panthéon-Sorbonne, HiCSA 